\title{
Short wave-automated perimetry (SWAP) versus optical coherence tomography in early detection of glaucoma
}

\author{
This article was published in the following Dove Press journal: \\ Clinical Ophthalmology \\ 19 September 2016 \\ Number of times this article has been viewed
}

Objective: To assess the role and diagnostic effectiveness of optical coherence tomography (OCT) and short wave-automated perimetry (SWAP) to distinguish between normal, glaucoma suspects, and surely diagnosed glaucomatous eye.

Background: Changes in the optic disc and retinal nerve fiber layer (RNFL) often precede the appearance of visual field defect with standard automated perimetry. Unfortunately, RNFL defect can be difficult to identify during clinical examination. Early detection of glaucoma is still controversial, whether by OCT, SWAP, or frequency-doubling technology perimetry.

Patients and methods: In this randomized controlled, consecutive, prospective study, a total 70 subjects (140 eyes) were included in the study, divided into three groups: Group A, 10 healthy volunteers (20 eyes); Group B, 30 patients (60 eyes) with glaucoma suspect; and Group C, 30 patients (60 eyes) with already diagnosed glaucomatous eyes.

Results: Average RNFL thickness was $75 \pm 9.0$ in the glaucoma group, $99 \pm 15.5$ in the control group, and $94 \pm 12$ in glaucoma suspect. The inferior quadrant was the early parameter affected. There was significant correlation between visual field parameters and RNFL thickness in both glaucoma and glaucoma suspect groups.

Conclusion: Both RNFL thickness measured by OCT and SWAP indices are good discrimination tools between glaucomatous, glaucoma suspect, and normal eyes. OCT parameters tend to be more sensitive than SWAP parameters.

Keywords: OCT, SWAP, glaucoma, intraocular pressure, RNFL

\section{Introduction}

Currently, glaucoma is defined as a disturbance of the structural or functional integrity of the optic nerve that causes characteristic atrophic changes in the optic nerve and retinal nerve fiber layer (RNFL) associated with visual field changes with or without increase in intraocular pressure (IOP). ${ }^{1}$ As this injury is largely irreversible, early detection of glaucomatous damage is visually important. Examination of optic nerve head and RNFL is necessary in both diagnosis and follow-up of glaucoma. ${ }^{2}$

Changes in the optic disc and RNFL often precede the appearance of visual field defect with standard automated perimetry (SAP). Unfortunately, RNFL defect can be difficult to identify during clinical examination as this is a subjective method needing clear optical media, highly efficient red free filter, and advanced defect. ${ }^{3,4}$ Therefore, objective investigations of measuring these structures facilitate ophthalmologists to reach to definite diagnosis.

Optical coherence tomography (OCT) is a noninvasive imaging modality that uses low-coherence light to obtain a high-resolution cross-section of anterior and
Correspondence: Adel Galal Zaky Department of Ophthalmology, Faculty of Medicine, Menoufia University, Yasen Abd EL Ghafar Street, Shebein El Kom, 325II, Menoufia, Egypt

Tel +201006185456

Email adelzaky12@gmail.com 
posterior segments of the eye and quantitative assessments of different layers. ${ }^{5}$

Short wave-automated perimetry (SWAP) is more sensitive to early glaucoma than standard perimetry. ${ }^{6}$ With SWAP, a large Goldmann size $\mathrm{V}$ blue target is projected against a bright yellow background. The background reduces the sensitivities of the green and red cones, thus isolating the short wavelength-sensitive blue cones and their associated small, bistratified retinal ganglion cells. ${ }^{6}$

The purposes of the study are to assess the role and diagnostic ability of OCT and SWAP to distinguish between normal, glaucoma suspects, and surely diagnosed glaucomatous eye.

\section{Patients and methods}

This consecutive prospective study was conducted on patients attending the outpatient's clinic of Menoufia University Hospital and Banha Teaching Hospital by the same operator during the period from January 2013 to October 2015.

A total of 70 subjects (140 eyes) were included in the study and divided into three groups: Group A, 10 healthy volunteers (20 eyes); Group B, 30 patients (60 eyes) with glaucoma suspect; and Group C, 30 patients (60 eyes) with already diagnosed glaucomatous eyes. Written informed patient consent was obtained.

\section{Selection criteria}

The selection criteria were as follows: Best-corrected visual acuity at least 6/12, with spherical refractive error between $+2 \mathrm{D}$ and $-2 \mathrm{D}$ and astigmatism $<2 \mathrm{D}$. All eyes had normal open angle.

\section{Exclusion criteria}

The exclusion criteria were as follows: Patients with any type of retinal pathology, history of retinal or refractive laser procedures, history of retinal surgery, patients with secondary glaucoma, history of diabetes, significant media opacity (as cataract), or pachymetry $>480 \mu \mathrm{m}$ or $<540 \mu \mathrm{m}$ were excluded. Patients with unreliable visual fields defined as false-negative $>33 \%$, falsepositive $>33 \%$, and fixation errors $>20 \%$ were also excluded.

All patients had a full ophthalmic examination including visual acuity, refraction, Goldman applanation tonometry, gonioscopy, pachymetry, dilated fundus examination, standard automated visual field examination, SWAP using Humphrey Field Analyzer (Carl Zeiss Meditec, Inc), and finally nerve fiber layer thickness measurement by OCT (Carl Zeiss Meditec, Inc).

Normal patients were normally health volunteers with negative family history for glaucoma. All of them had IOP less than $21 \mathrm{mmHg}$, with absence of glaucomatous optic neuropathy (GON) with normal visual field indices.

Glaucoma suspects either had (normal tension, normal visual fields with glaucomatous optic neuropathy) or (normal visual fields, absent glaucomatous optic neuropathy and high IOP higher than $21 \mathrm{mmHg}$ ).

Glaucomatous eyes show GON associated with characteristic visual field changes in the corresponding hemi field.

Glaucomatous eyes were subdivided according to the deviation of SAP (Hodapp's classification) ${ }^{7}$ into three subgroups. Early was defined by visual field loss with an $\mathrm{MD}<-6 \mathrm{~dB}$, moderate glaucoma with MD between -6 and $-12 \mathrm{~dB}$, and severe glaucoma with MD worse than $-12 \mathrm{~dB}$.

\section{Optical coherence tomography}

The OCT Cirrus (Carl Zeiss Meditec, Inc, Dublin, CA, USA) employs a low-coherence interferometer to assess peripapillary tissue thickness. A good quality image was defined as an image with signal-to-noise ratio less than $50 \mathrm{~dB}$. By using inbuilt RNFL thickness average analysis, mean RNFL thickness can be measured. ${ }^{8}$

A functional disc map was made by dividing the optic nerve head into 12 equal radial sectors, each one of them for $30^{\circ}$.

Three different parameters were performed for RNFL thickness:

1. The average thickness of RNFL for the entire circumference of the optic disc.

2. RNFL thickness in each sector (clock hours).

3. The thickness at each quadrant $\left(90^{\circ}\right)$, namely, superior $\left(46^{\circ}-135^{\circ}\right)$, nasal $\left(136^{\circ}-225^{\circ}\right)$, inferior $\left(226^{\circ}-315^{\circ}\right)$, and temporal $\left(316^{\circ}-45^{\circ}\right)$.

\section{Short wave-automated perimetry (SWAP) and standard automated perimetry (SAP)}

SWAP is a modification of SAP using Humphrey Field Analyzer 745i (Zeiss Humphrey Systems, Dublin, CA, USA). It utilizes a $440 \mathrm{~nm} 1.8^{\circ}$ target at 200 milliseconds duration on a 100 candelas $/ \mathrm{m}^{2}$ yellow background to selectively test the short wave length-sensitive cones and their connection. First field done to any patient was discarded, whether white/white (W/W) or SWAP. Testing was done in more than one session to prevent the effects of patient's fatigue. No more than two fields were done on one single visit, and no more than one SWAP testing was done per day. Patient visits were separated by duration of at least a week. All visual fields that were used for analysis satisfied the following reliability criteria: fixation losses $<25 \%$ and false-positive and false-negative responses $<20 \%$. For each reliable field, 
mean deviation (MD) and pattern standard deviation (PSD) were recorded.

Results were collected, tabulated, and statistically analyzed using the IBM personal computer and Statistical Package for the Social Sciences (SPSS, Chicago, IL, USA) version 16. In the statistical comparison between the different groups, the significance of difference was tested using one of the following tests:

1. Student's $t$-test and Mann-Whitney test: used to compare mean of two groups of quantitative data of parametric and nonparametric, respectively.

2. Analysis of variance test ( $F$ value): used to compare mean of more than two groups of quantitative data. Intergroup comparison of categorical data was performed by using chi-square test ( $\chi^{2}$-value) and Fisher's exact test (FET).

3. Correlation coefficient: to find relationships between variables. A $P$-value $<0.05$ was considered statistically significant $(\mathrm{S})$, while a value $>0.05$ was statistically insignificant. A $P$-value $<0.01$ was considered highly significant in all analyses.

\section{Results}

A total of 70 patients (140 eyes) were included in the study. Their demographic features are given in Table 1. Regarding age and sex there were no statistically significant differences between the groups, but female sex was found to be a significant factor. RNFL thickness values in all parameters (average and quadrants) measured by OCT are listed in Table 2. RNFL thickness was greatest in the superior and inferior quadrants in the control group. RNFL thickness in the nasal and temporal quadrants was significantly thinner than in superior and inferior quadrants, demonstrating the so-called double-hump pattern.

MD and PSD of SAP and SWAP are listed in Table 3.

In the glaucoma suspect group, RNFL thickness measured by OCT was outside normal limits in at least 1 hour in 7 eyes. Twenty-one eyes (35\%) were following ISNT rule while the other 39 eyes were against (65\%). Forty-three eyes of $60(71.7 \%)$ had IOP equal to or higher than $21 \mathrm{mmHg}$ at least in two different times with no GON. Twenty-one eyes of 43 (35\%) had glaucomatous field changes in SWAP (generalized depressed, paracenteral scotomas, arcuate scotomas, nasal step, temporal wedge, and altitudinal defect). Seventeen eyes of 60 (28.3\%) had GON with normal IOP. These 17 eyes (28.3\%) had glaucomatous field changes in SWAP (generalized depressed, paracenteral scotomas, arcuate scotomas, nasal step, temporal wedge, and altitudinal defect). Thirty-eight eyes $(63.3 \%)$ in this group had glaucomatous field changes in SWAP (generalized depressed, paracenteral scotomas, arcuate scotomas, nasal step, temporal wedge, and altitudinal defect).

In glaucoma group, RNFL thickness measured by OCT was outside normal limits in at least 1 hour in 9 eyes. Fortythree eyes (71.7\%) did not comply with the ISNT rule, while 17 eyes $(28.3 \%)$ did. All the 60 eyes had glaucomatous field changes in SAP (generalized depressed, paracenteral scotomas, arcuate scotomas, nasal step, temporal wedge, and altitudinal defect). Fifty-two eyes of 60 (86.7\%) had corresponding OCT findings (RNFL thickness defect), while 8 eyes $(13.3 \%)$ did not have this corresponding OCT findings. All of the 52 eyes showed similar but more advanced and denser glaucomatous field changes in SWAP correlated to these finding in SAP.

\section{Correlation between RNFL thickness by OCT and SWAP in glaucoma group}

Average RNFL thickness showed a stronger correlation (highly statistically significant $P=0.001$ ) with SWAP MD (0.387) than that was found between average RNFL thickness and PSD (0.111) of SWAP $(P=0.009)$. Inferior quadrant thickness showed a highly significant statistically correlation with MD (0.563) and PSD (0.185; $P=0.001)$.

Superior quadrant thickness showed a highly statistically significant correlation with SWAP (MD 0.19; $P=0.001)$, while there was no significant correlation with $\operatorname{PSD}(P=0.094)$.

Table 4 shows that the strongest correlation in this group was found between RNFL inferior quadrant thickness and SWAP MD (0.563), which was found to be highly statistically significant $P=0.001$.

Table I Demographic features among three groups

\begin{tabular}{|c|c|c|c|c|c|}
\hline Variable & Case group & Suspect group & Control group & $F$ test & $P$-value \\
\hline \multicolumn{6}{|l|}{$\overline{\text { Age }}$} \\
\hline Mean \pm SD & $55.32 \pm 9.22$ & $43.7 \pm 12.92$ & $44.95 \pm 9.76$ & 18.09 & 0.001 \\
\hline \multicolumn{6}{|l|}{ Sex, n (\%) } \\
\hline Male & $20(33.3)$ & $20(33.3)$ & $10(50.0)$ & $\mathrm{FET}=37.93$ & 0.001 \\
\hline Female & $40(66.7)$ & $40(66.7)$ & $10(50.0)$ & $\mathrm{FET}=37.93$ & 0.001 \\
\hline
\end{tabular}

Abbreviation: FET, Fisher's exact test. 
Table 2 RNFL thickness in (four quadrants, average, and sup/inf difference) among three groups

\begin{tabular}{|c|c|c|c|c|c|}
\hline Variable & Case group & Suspected group & Control group & $F$ test & $P$-value \\
\hline Superior mean $\pm S D$ & $95.17 \pm 21.92$ & $114.35 \pm 12.62$ & $126.0 \pm 10.48$ & 32.29 & 0.001 \\
\hline Inferior mean \pm SD & $105.4 \pm 25.05$ & $117.35 \pm 18.37$ & $128.0 \pm 1 \mid .53$ & 10.39 & 0.001 \\
\hline Nasal mean \pm SD & $66.3 \pm 12.49$ & $65.75 \pm 6.9$ & $89.5 \pm 7.71$ & 49.29 & 0.001 \\
\hline Temporal mean \pm SD & $63.45 \pm 15.95$ & $62.05 \pm 14.12$ & $70.6 \pm 8.19$ & 2.72 & 0.069 \\
\hline Sup/inf difference mean $\pm S D$ & $10.23 \pm 25.25$ & $3.0 \pm 17.24$ & $2.0 \pm 11.96$ & 2.29 & 0.105 \\
\hline Average thickness & $75 \pm 9.0$ & $94 \pm 12$ & $99 \pm 15.5$ & 11.56 & 0.001 \\
\hline
\end{tabular}

Abbreviations: RNFL, retinal nerve fiber layer; sup, superior; inf, inferior.

\section{Correlation between RNFL thickness by OCT and SWAP in glaucoma suspect group}

Average RNFL thickness showed a stronger correlation (highly statistically significant $P=0.001$ ) with SWAP MD (0.171) than that was found between average RNFL thickness and PSD ( 0.145$)$ of SWAP $(P=0.007)$.

Inferior and superior quadrants thickness showed a highly statistically significant correlation with $\mathrm{MD}(0.252$ and 0.173 ; $P=0.001)$ and $\operatorname{PSD}(0.416$ and $0.134 ; P=0.001$ and 0.004$)$.

Nasal quadrant thickness showed a highly statistically significant correlation with SWAP (MD 0.113; $P=0.003$ ).

Table 5 shows that the strongest correlation in this group was found between RNFL inferior quadrant thickness and SWAP PSD (0.416), and this was $P=0.001$, highly statistically significant.

\section{Discussion}

Primary open-angle glaucoma is currently incurable, but glaucoma subspecialists agree that early diagnosis and treatment are essential for controlling the disease and reducing vision loss. The diagnosis of glaucoma requires a multifaceted analysis of patients' history and clinical findings, including assessments of visual function and imaging studies. ${ }^{9}$

OCT is considered as an objective investigative tool that provides quantitative information about RNFL thickness. In addition, there are no effects of refractive error or corneal birefringence on OCT information. ${ }^{10}$

In our study, there was significant difference in RNFL thickness among control group, glaucoma, and glaucoma suspected. Previous studies have shown that RNFL thickness measured by OCT is a good tool for differentiating glaucomatous from normal eyes. ${ }^{11}$ Chen et al ${ }^{12}$ showed that average RNFL thickness was the best parameter for differentiating glaucoma from normal eyes. Sibota and Sony ${ }^{11}$ found that average RNFL thickness followed by inferior RNFL thickness had the highest power to discriminate between glaucomatous and normal eyes. ${ }^{11}$ In recent studies, Chen \& Huang showed that inferior RNFL was the best parameter for differentiation. ${ }^{12}$

Results in our study showed that OCT and short-wavelength perimetry were well correlated. There was a statistically significant correlation between MD, PSD, and both average RNFL thickness ( 0.622 and -0.334 ; $P=0.001$ and 0.009 ) and inferior quadrant among the glaucoma group $(0.75$ and $-0.431 ; P=0.001)$. However, superior RNFL thickness significantly correlated only with MD (0.436; $P=0.001)$.

We also found that among the glaucoma suspect group there was a statistically significant correlation between MD, PSD as visual field indices, and inferior quadrant $(-0.502$ and $0.645 ; P=0.001$ ), followed by average RNFL thickness $(-0.413$ and $0.381 ; P=0.001$ and 0.003$)$, and finally with superior quadrant $(-0.417$ and $0.366 ; P=0.001$ and 0.004$)$. However, nasal RNFL thickness significantly correlated only with MD $(-0.377 ; P=0.003)$.

Vishva and Glen ${ }^{13}$ showed that structure-function relationship was enhanced with RNFL thickness as the correlation $R^{2}$ between RNFL thickness and visual field indices ranged from $5 \%$ nasal to $25 \%$ inferotemporal and was

Table 3 Visual field parameters among glaucoma and glaucoma suspect group

\begin{tabular}{lllll}
\hline Variable & Case group & Suspected group & Test & P-value \\
\hline SWAP MD mean \pm SD & $-8.67 \pm 7.69$ & $-4.47 \pm 2.95$ & $Z=2.95$ & 0.003 \\
SWAP PSD mean \pm SD & $3.88 \pm 1.62$ & $2.84 \pm 0.59$ & $t=4.73$ & 0.001 \\
SAP MD mean \pm SD & $-7.95 \pm 9.93$ & $-3.06 \pm 2.94$ & $Z=2.81$ & 0.005 \\
SAP PSD mean \pm SD & $3.34 \pm 2.4$ & $2.16 \pm 1.16$ & $t=3.43$ & 0.001 \\
\hline
\end{tabular}

Abbreviations: SWAP, short wave-automated perimetry; MD, mean deviation; PSD, pattern standard deviation; SAP, standard automated perimetry. 
Table 4 Correlation between SWAP PSD, SWAP MD, and RNFL thickness among glaucoma group

\begin{tabular}{llllll}
\hline Case group & \multicolumn{2}{l}{ SWAP MD } & & \multicolumn{2}{l}{ SWAP PSD } \\
\cline { 2 - 3 } & $\boldsymbol{R}$ & $\boldsymbol{P}$-value & & $\boldsymbol{R}$ & $\boldsymbol{P}$-value \\
\hline RNFL thickness & 0.387 & 0.001 & & $0.11 \mathrm{I}$ & 0.009 \\
Superior Q OCT & 0.19 & 0.001 & & 0.047 & 0.094 \\
Inferior Q OCT & 0.563 & 0.001 & & 0.185 & 0.001 \\
Nasal Q OCT & 0.005 & 0.589 & & 0.011 & 0.427 \\
Temporal Q OCT & 0.062 & 0.055 & & 0.003 & 0.884 \\
\hline
\end{tabular}

Abbreviations: SWAP, short wave-automated perimetry; MD, mean deviation PSD, pattern standard deviation; OCT, optical coherence tomography; RNFL, retinal nerve fiber layer.

relatively stronger than other neuroretinal rim measurement and was not significantly different from that with Bruch's membrane opening-based horizontal rim width.

In addition, Sibota et $\mathrm{al}^{11}$ found a correlation between visual field parameters (MD and PSD) and the average RNFL thickness. There was a significant positive correlation with MD and a significant negative correlation with PSD.

The same holds good for Yalvac and Altunsay, ${ }^{14}$ who found a significant correlation between global indices of visual field MD, PSD, and RNFL thickness. However, Paris ${ }^{15}$ found a highly significant correlation between average RNFL thickness and PSD. He also showed a less significant correlation between MD and RNFL thickness.

In this study, we found that MD measured by SWAP is lower than that measured by SAP, and PSD measured by SWAP is higher than that measured by SAP. We also found that 38 eyes $(63.3 \%)$ in the glaucoma suspect group that had glaucomatous field changes in SWAP (generalized depressed, paracentral scotomas, arcuate scotomas, nasal step, temporal wedge, and altitudinal defect) were free by SAP.

Sample and Weinreb ${ }^{16}$ followed 25 eyes with ocular hypertension for 13-37 months. Later, five eyes showed glaucoma based on the results of a repeatable visual field defect by SWAP; of these, four were classified as high risk and one as medium risk.

Table 5 Correlation between SWAP PSD, SWAP MD and other variables among suspected group

\begin{tabular}{llllll}
\hline Suspected group & \multicolumn{2}{c}{ SWAP MD } & & \multicolumn{2}{c}{ SWAP PSD } \\
\cline { 2 - 3 } \cline { 6 - 7 } & $\boldsymbol{R}$ & $\boldsymbol{P}$-value & & $\boldsymbol{R}$ & $\boldsymbol{P}$-value \\
\hline RNFL thickness & 0.171 & 0.001 & & 0.145 & 0.007 \\
Superior Q OCT & 0.173 & 0.001 & & 0.134 & 0.004 \\
Inferior Q OCT & 0.252 & 0.001 & & 0.416 & 0.001 \\
Nasal Q OCT & 0.113 & 0.003 & & 0.035 & 0.148 \\
Temporal Q OCT & 0.01 & 0.937 & & 0.014 & 0.356 \\
\hline
\end{tabular}

Abbreviations: SWAP, short wave-automated perimetry; MD, mean deviation; PSD, pattern standard deviation; OCT, optical coherence tomography; RNFL, retinal nerve fiber layer.

\section{Conclusion}

Both RNFL thickness measured by OCT and SWAP indices are good discrimination tools between glaucomatous, glaucoma suspect, and normal eyes. There is superior ability of SWAP over SAP in detecting glaucomatous changes in glaucoma suspect group.

\section{Disclosure}

The authors report no conflicts of interest in this work.

\section{References}

1. Sommer A, Miller NR, Pollack, Maumenee AE, George T. The nerve fiber layer in the diagnosis of glaucoma. Arch Ophthalmol. 1977;95: 2149-2156.

2. Quigley HA, Miller NR, Gearge T. Clinical evaluation of nerve fiber layer atrophy as an indicator of glaucomatous optic nerve damage. Arch Ophthalmol. 1980;98:1564-1571.

3. Johnson CA, Samole PA, Zangwill LM. Structure and function evaluation (SAFE): comparison of optic disc and visual field characteristics. Am J Ophthalmol. 2003;135:148-154.

4. Kass MA, Heuer DK, Higginbotham EJ. The ocular hypertension treatment study: a randomized trial determines that topical ocular hypotensive medications delays or prevents the onset of primary open angle glaucoma. Arch Ophthalmol. 2002;120:701-713.

5. Bowd C, Weinreb RN, Williams JM. The retinal nerve fiber layer thickness in ocular hypertensive, normal and glaucomatous eyes with optical coherence tomography. Arch Ophthalmol. 2000;118:22-26.

6. Medeiros FA, Sample PA, Weinreb RN. Corneal thickness measurements and visual function abnormalities in ocular hypertensive patients. Am J Ophthamol. 2013;135(2):131-137.

7. Hodapp E, Parish RK, Anderson DR. Clinical Decisions in Glaucoma. St. Louis: C.V. Mosby; 1993:84-125.

8. Kiernan DF, Mieler WF, Hariprasad SM. Spectral-domain optical coherence tomography: a comparison of modern high-resolution retinal imaging systems. Am J Ophthalmol. 2010;149(1):18-31.

9. Medeiros FA, Alencar LM, Zangwill LM. Prediction of functional loss in glaucoma from progressive optic disc damage. Arch Ophthalmol. 2009;127:1250-1256.

10. Bawd C, Zangwill LM, Berry CC. Detecting early glaucoma by assessment of retinal nerve fiber thickness and visual function. Invest Ophthalmol Vis Sci. 2008;42:1993-2003.

11. Sibota R, Sony P. Diagnostic capability of optical coherence tomography in evaluating the degree of glaucomatous retinal nerve fiber damage. Invest Ophthalmol Vis Sci. 2006;47:2006-2010.

12. Chen HY, Huang KI. Discrimination between normal and glaucomatous eyes using optical coherence tomography in Taiwan Chinese subjects. Graefes Arch Clin Expophthalmol. 2005;243:894-902.

13. Vishva M, Glen P. Enhanced structure-function relationship in glaucoma with an anatomically and geometrically accurate neuroretinal rim measurement. Invest Ophthalmol Vis Sci. 2015;56:98-105.

14. Yalvac Hs, Altunsay M. The correlation between visual field defects and focal nerve fiber layer thickness measured with optical coherence tomography in evaluation of glaucoma. J Glaucoma. 2009;18:53-61.

15. Paris V. Correlation between optical coherence tomography, pattern electro retinogram and visual evoked potentials in open angle glaucoma. Ophthalmology. 2010;108:905-912.

16. Sample PA, Weinreb RN. Corneal thickness measurements and visual function abnormalities in ocular hypertensive patients. Am J Ophthalmol. 2003;135:131-137. 


\section{Publish your work in this journal}

Clinical Ophthalmology is an international, peer-reviewed journal covering all subspecialties within ophthalmology. Key topics include: Optometry; Visual science; Pharmacology and drug therapy in eye diseases; Basic Sciences; Primary and Secondary eye care; Patient Safety and Quality of Care Improvements. This journal is indexed on

Submit your manuscript here: http://www.dovepress.com/clinical-ophthalmology-journal
PubMed Central and CAS, and is the official journal of The Society of Clinical Ophthalmology (SCO). The manuscript management system is completely online and includes a very quick and fair peer-review system, which is all easy to use. Visit http://www.dovepress.com/ testimonials.php to read real quotes from published authors. 